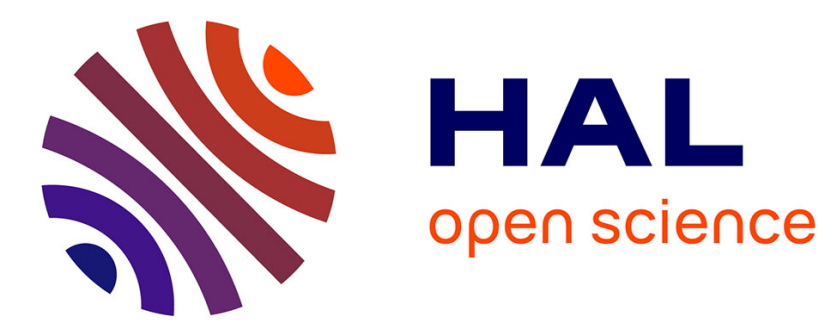

\title{
Scattering correction using continuously thickness-adapted kernels
}

Navnina Bhatia, David Tisseur, Fanny Buyens, Jean Michel Létang

\section{To cite this version:}

Navnina Bhatia, David Tisseur, Fanny Buyens, Jean Michel Létang. Scattering correction using continuously thickness-adapted kernels. NDT \& E International, 2016, 78, pp.52-60. 10.1016/j.ndteint.2015.11.004 . hal-01272912

\section{HAL Id: hal-01272912 \\ https://hal.science/hal-01272912}

Submitted on 7 Jan 2019

HAL is a multi-disciplinary open access archive for the deposit and dissemination of scientific research documents, whether they are published or not. The documents may come from teaching and research institutions in France or abroad, or from public or private research centers.
L'archive ouverte pluridisciplinaire HAL, est destinée au dépôt et à la diffusion de documents scientifiques de niveau recherche, publiés ou non, émanant des établissements d'enseignement et de recherche français ou étrangers, des laboratoires publics ou privés. 


\title{
Scattering correction using continuously thickness-adapted kernels
}

\author{
Navnina Bhatia ${ }^{\mathrm{a}, *}$, David Tisseur ${ }^{\mathrm{a}}$, Fanny Buyens ${ }^{\mathrm{a}}$, Jean Michel Létang ${ }^{\mathrm{b}}$ \\ ${ }^{a}$ CEA, LIST, F-91191, Gif-sur-Yvette, France \\ ${ }^{\mathrm{b}}$ Université de Lyon, CREATIS, CNRS UMR5220, INSERM U1044, Université Claude Lyon 1, Centre Léon Bérard, France
}

\begin{abstract}
Quantitative reconstruction values are often miscalculated in Cone Beam Computed Tomography (CBCT) due to the presence of secondary radiation originating from scattering of photons inside the object and detector under consideration. The effect becomes more prominent and challenging in case of X-ray source of high energy (over a few $100 \mathrm{keV}$ ) which is used in industrial Non-Destructive Testing (NDT), due to higher scatter to primary ratio (SPR). This paper describes a scatter correction algorithm for correcting the combined scattering due to the object and the detector based on variations in Scatter Kernel Superposition (SKS) method. Scatter correction is performed for homogeneous and heterogeneous objects in a robust iterative manner suitable for high SPR, using pencil beam kernels which are simulated in computed tomography (CT) module of the CIVA software for NDT simulations. Two methods for scatter correction using SKS approach are discussed and compared in the paper. In the first method, we use a discrete approach in which kernels for only few thicknesses are used. In the second method a continuous approach is proposed where the kernels are analytically parameterised for all thicknesses. The results obtained after scatter correction are well within the expected reconstruction values. The continuous method produces better edge enhanced corrected projections and the method results in improved reconstruction values than the discrete method.
\end{abstract}

\section{Introduction}

Compared to highly collimated fan beam computed tomography (CT), cone-beam computed tomography ( $\mathrm{CBCT}$ ) is based on 2D detectors of larger area, which makes CBCT bear a high level of $\mathrm{X}$-ray scatter. Inadequate modeling of this scatter leads to cupping and streaking artifacts [1] and to a global degradation of image quality in CBCT.

There are various existing CBCT scatter correction methods which can be summed up mainly into two categories: pre-processing methods, and post-processing methods. Pre-processing methods modify the $\mathrm{X}$-ray system and are able to separate the scatter from the primary photons based on the difference of their incidence angles, but require a higher dose. These include anti-scatter grids method (which make use of highly attenuating grids which are mounted directly on the top of the detector) [2] and the airgap method [3]. The post-processing methods estimate the scatter signal from the scatter-contaminated projection using some prior knowledge of the scatter distribution. These include measurement based methods such as beam stop array [4,5] where a high atomic number material like lead (strip or a disc) is inserted between the X-ray source and the imaged object. Such methods cause

\footnotetext{
*Corresponding author. Tel.: +33169087863.

E-mail address: navnina.bhatia@cea.fr (N. Bhatia).
}

increased X-ray exposure due to more than one scans per projection, prolong the scanning time and are also subject to error due to object motion. Many other software based post-processing have been proposed for scatter estimation and correction [6,7].

In this paper we propose to focus on Scatter Kernel Superposition (SKS) method [8-11] in which the scatter signal is modeled as the sum of the scatter contributions from a group of pencil beams passing through the object. It approximates the scatter distribution, as the convolution of primary signal with scatter kernels. This method requires no additional hardware, scanning time and additional dose. Pencil beam kernels are thickness dependent kernels and there is an appreciable change in the amplitude and shape of these kernels with respect to small variation in the thickness of the object. The scatter correction methods are based on a discrete set of thickness-dependent kernels and for a range of thickness only one kernel is used. This method gives satisfactory results in many applications. However, when a high range is considered (typically [300,500] keV), the SPR is expected to be very large and the different steps of the SKS correction algorithm have to be reconsidered. In particular a better sampling of the kernels with respect to the thickness of the object is required to get an accurate model of variability in shape and in amplitude of the scatter kernels over the whole thickness range. When the scatter level is not negligible with respect to the primary radiations, the robustness and the convergence of the 
iterative correction scheme become critical since a slight overestimation of the scatter radiation level might lead to large negative values of the primary transmittance.

We propose in this paper a twofold modification of Sun and Starlack [11] SKS approach to tackle X-ray imaging with larger SPR: an analytical parameterization of the scatter kernel is derived in terms of material thickness and a multiplicative iteration approach is implemented. We begin by describing the methodology of scatter correction with pencil beam kernels. This is followed by the description of generation of kernels and development of the analytic expression for continuous kernel map. The modeling of the kernels for heterogeneous object is described in detail. We then describe the iterative scheme followed for the scatter correction. Afterwards the acquisition set up and objects used are described in detail. Finally the results obtained after the reconstruction performed using FDK algorithm are compared for discrete set of kernels and continuous map of kernels for homogeneous and heterogeneous object.

\section{Method and materials}

\subsection{Scatter correction using pencil beam kernels}

The measured signal at the detector $I(m, n)$ has two components: $P(m, n)$ is the primary signal contributed by the photons passing directly without any attenuation or scattering and $S(m, n)$ is the signal contribution of the scattered photons from the object and the detector. Therefore, the measured signal is given by:

$I(m, n)=P(m, n)+S(m, n)$

where $m$ and $n$ correspond to the pixel position on the detector.

The scatter signal can be modeled as the sum of the scatter contributions from a group of pencil beams passing through the object and the detector. For each pencil beam input, a resulting kernel which has the weight of the scatter to primary ratio is determined. The total scatter signal $S(m, n)$ can then be modeled as:

$S(m, n)=\sum_{k} \sum_{l} P(k, l) h_{T(k, l)}(m-k, n-l)$

where, $h_{T}$ is the thickness $(T)$ dependent kernel, with amplitude equal to the ratio of the scattered signal at the current pixel to the primary signal, at the pencil beam centered pixel. The thickness is calculated with the Beer Lambert law

$T(k, l) \approx \frac{1}{\mu} \ln \frac{O(k, l)}{P(k, l)}$

with $\mu$ being the attenuation constant of the object under consideration at the mean energy of the spectrum used. The pencil beam kernel $h_{T}$ can be fitted into the equation formed by an amplitude factor $C(k, l)$ (which is a function of the primary signal $P(m, n)$ and the un-attenuated air intensity $O(m, n))$ and a formfunction $G(m-k, n-l)$ consisting of two circularly symmetric Gaussian functions describing the shape of the kernel:

$h_{T}(m-k, n-l)=C(k, l) G(m-k, n-l)$

$C(k, l)=\left(\frac{P(k, l)}{O(k, l)}\right)^{\alpha} \ln \left(\frac{O(k, l)}{P(k, l)}\right)^{\beta}$

$\left.\left.G(m-k, n-l)=A \exp -\frac{(m-k)^{2}+(n-l)^{2}}{2 \sigma_{1}^{2}}\right)+B \exp -\frac{(m-k)^{2}+(n-l)^{2}}{2 \sigma_{2}^{2}}\right)$

Eq. (2) then becomes

$S(m, n)=\sum_{k} \sum_{l} P(k, l) C(k, l) G(m-k, n-l)$
In the discrete kernel approach, a few thickness ranges are selected from zero to maximum thickness of the object and a single average value for fitting parameters $\alpha, \beta, A, B, \sigma_{1}, \sigma_{2}$ is obtained for one particular thickness range. The superposition convolution equation is thus modified to the form

$S(m, n)=\sum_{i} \sum_{k} \sum_{l} P(k, l) R_{i}(k, l) C_{i}(k, l) G_{i}(m-k)(n-l)$

$R_{i}(k, l)= \begin{cases}1, & \text { if } T_{i}(k, l) \leq T(k, l)<T_{i+1}(k, l) \\ 0, & \text { otherwise }\end{cases}$

where $i$ gives the number of thickness groups and $T_{i}$ and $T_{i+1}$ are the lower and upper bound thicknesses of the $i$ th group.

In the continuous approach, the fitting parameters are also interpolated with respect to the thicknesses to form a continuously varying profile of kernels. Hence Eq. (8) is modified to:

$S(m, n)=\sum_{k} \sum_{l} P(k, l) C(k, l, T(k, l)) G(m-k, n-l, T(k, l))$

\subsection{Generation and fitting of kernels}

Monte Carlo (MC) simulations were performed in the CT module of CIVA software [12] for the generation of kernels. Developed by CEA LIST, CT module of CIVA combines deterministic and MC approach for the generation of primary and secondary radiation in tomography [13].

For the simulation of the kernels, imaging geometry corresponding to the acquisition set up was modeled in CIVA. Pencil beam source was impinged on slabs of same material as the object under study and discrete set of point spread 2D kernels were obtained on the flat panel detector. Eq. (4) was fit on these kernels

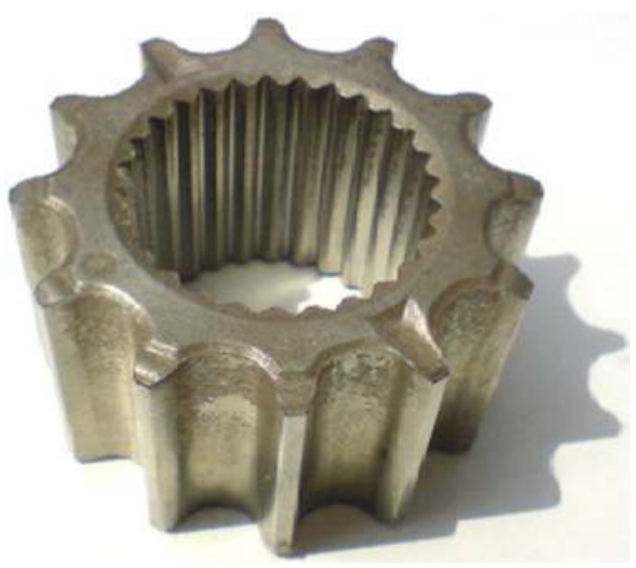

Fig. 1. Picture of sample of the iron hub [5].

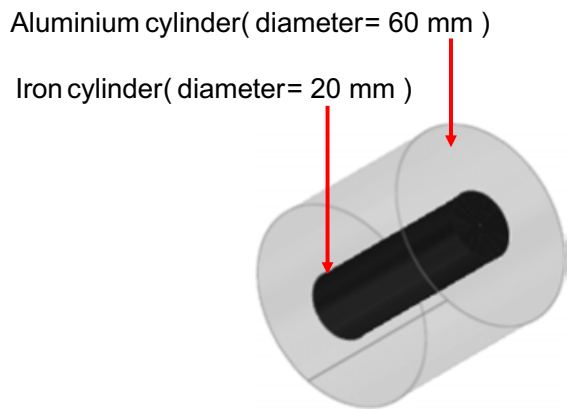

Fig. 2. Set-up of heterogeneous object consisting of a $60 \mathrm{~mm}$ diameter cylinder of aluminum containing a $20 \mathrm{~mm}$ diameter cylinder of iron. 

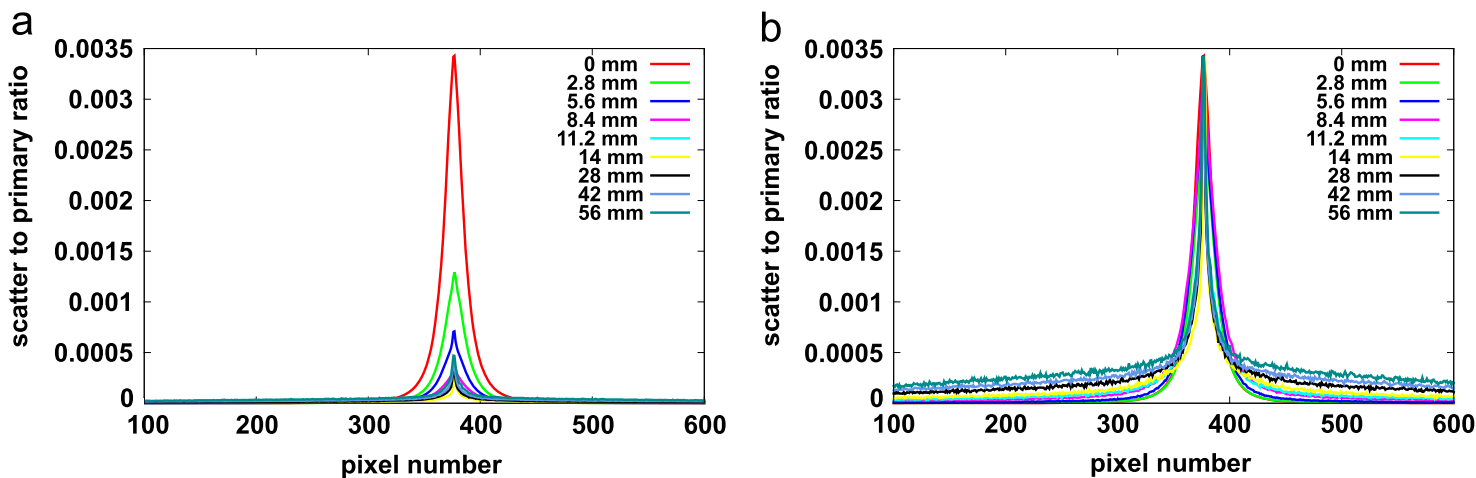

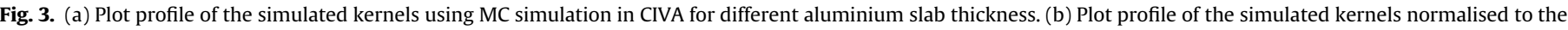
maximum value of kernel for detector kernel ( $0 \mathrm{~mm}$ thickness).
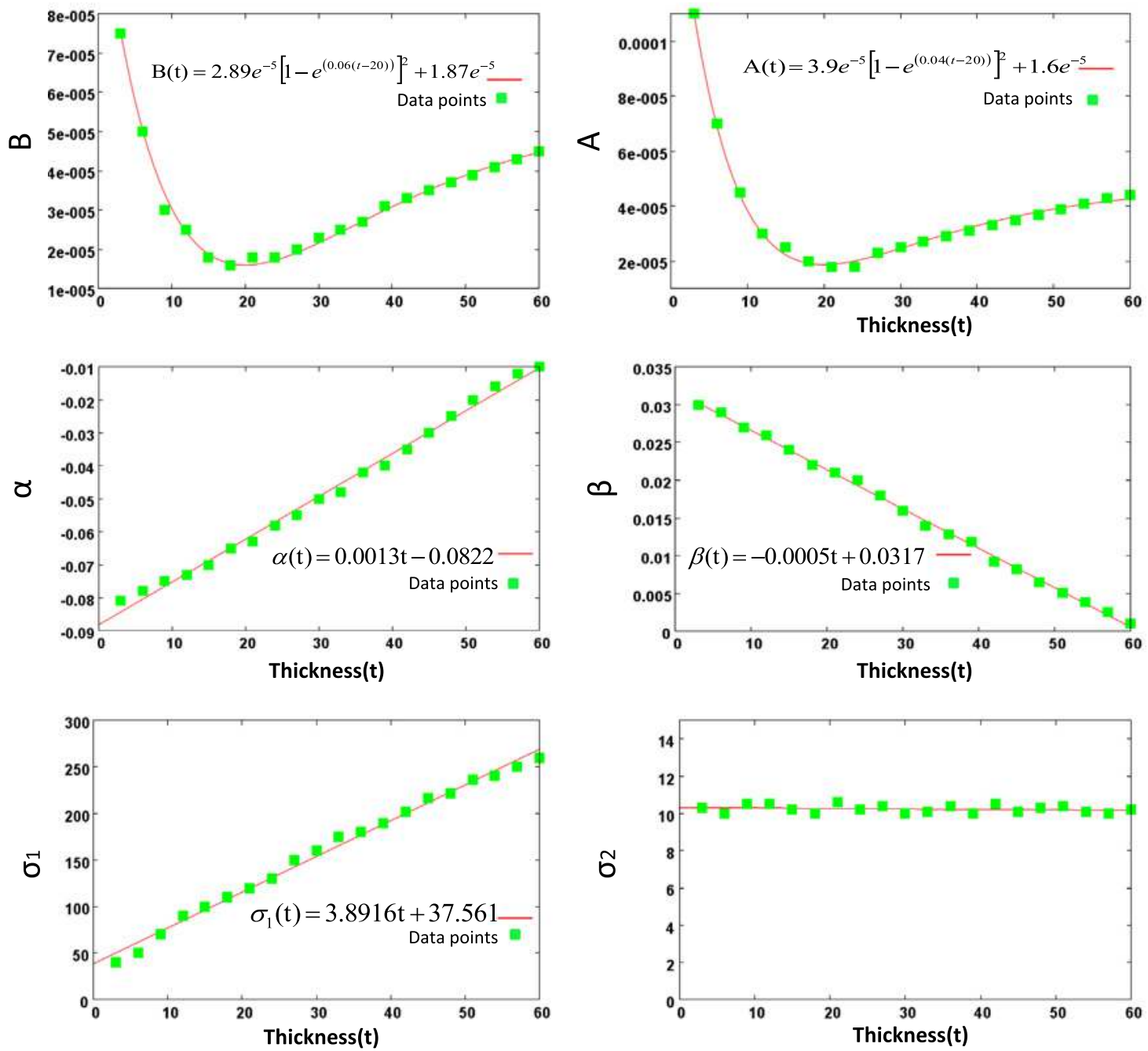

Fig. 4. Fitting of parameters $\alpha, \beta, A, B, \sigma_{1}, \sigma_{2}$ with thickness.

using non-linear least square fitting and the values for parameters $\alpha, \beta, A, B, \sigma_{1}, \sigma_{2}$ were calculated for these discrete sets of kernels.

To obtain the continuous kernel map, we analytically calculated the expression for the parameters $\alpha, \beta, A, B, \sigma_{1}, \sigma_{2}$ in terms of the thickness of the object. To obtain these expressions, the values of the parameters obtained for discrete set of kernels were fitted with respect to the thickness using non-linear least square curve fitting technique. The analytical models for the kernels parameters with respect to the material thickness are polynomials and Morse potential functions, with different constrain on the degrees of freedom for some. The two point-spread functions (PSF) in Eq. (6) are defined by standard deviations $\sigma_{1}$ and $\sigma_{2}$. These two PSF are representative of scattering of object and detector

1. $\sigma_{1}$ defines the higher order scattering from the object which is expected to increase with increasing the thickness of the object. 

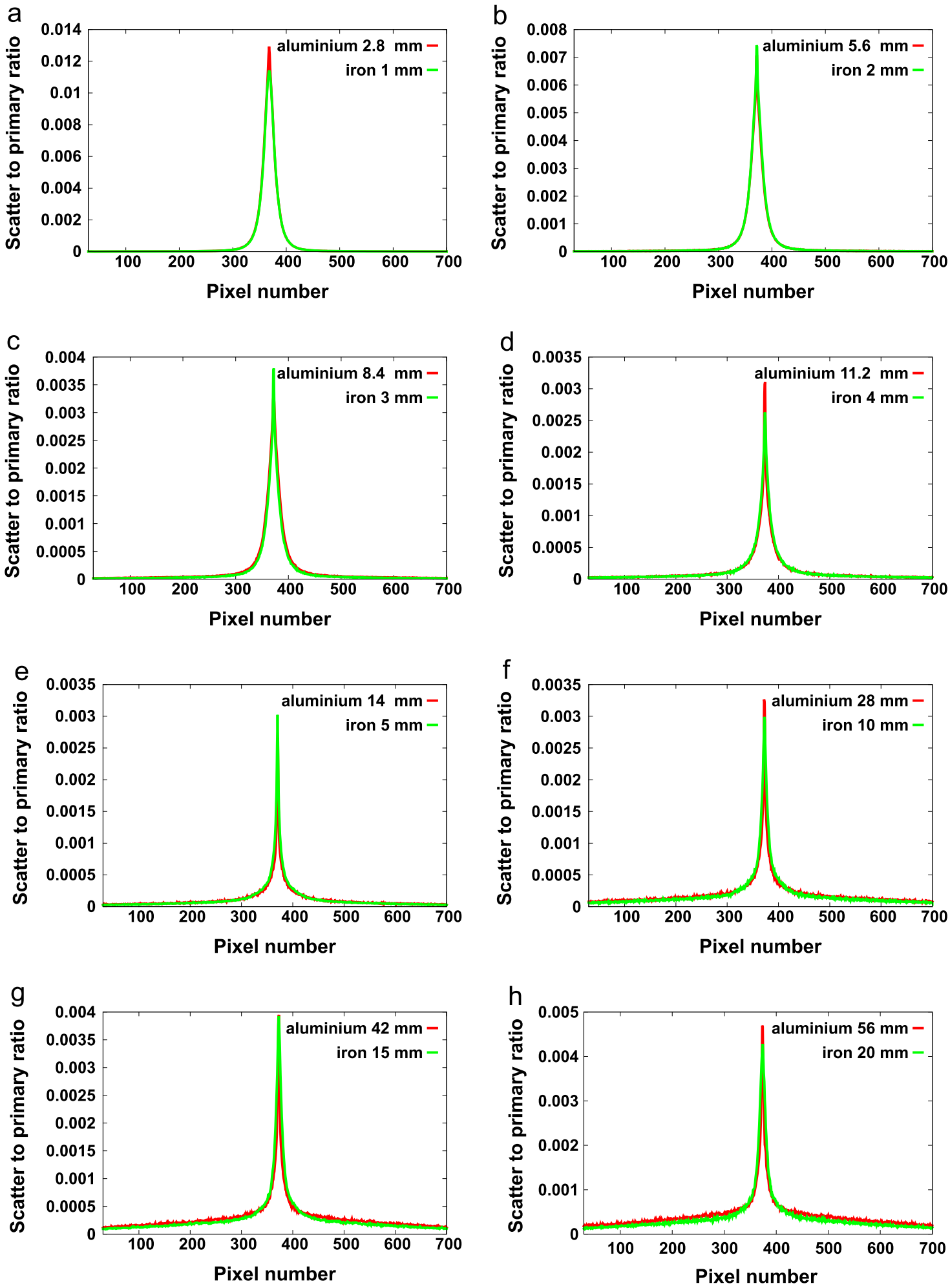

Fig. 5. Comparison of aluminum slab kernels with equivalent thickness iron slab kernels.

2. $\sigma_{2}$ defines the first order scattering dominated from the detector and is expected to be a constant with varying the thickness of the object.

\subsection{Modelling for heterogeneous objects}

When dealing with heterogeneous samples, the thickness map $T$ $(k, l)$ computed from Eq. (3) gives an estimate of the kernel-material equivalent thickness, that sometimes is called WET (water-equivalent thickness) when water is used to compute the kernels [10]. Within the energy range used, Compton scattering is the most dominant interaction process. The likelihood of interaction is proportional to electron density and is independent of the atomic number of the material. Therefore equivalent thickness $\left(T_{A}\right)$ of one material $\mathrm{A}$, for a given thickness $\left(T_{B}\right)$ of material $\mathrm{B}$, can be calculated in terms of their electron density functions using the following relationship:

$T_{A} \cdot \rho_{e, A}=T_{B} \cdot \rho_{e, B}$ 

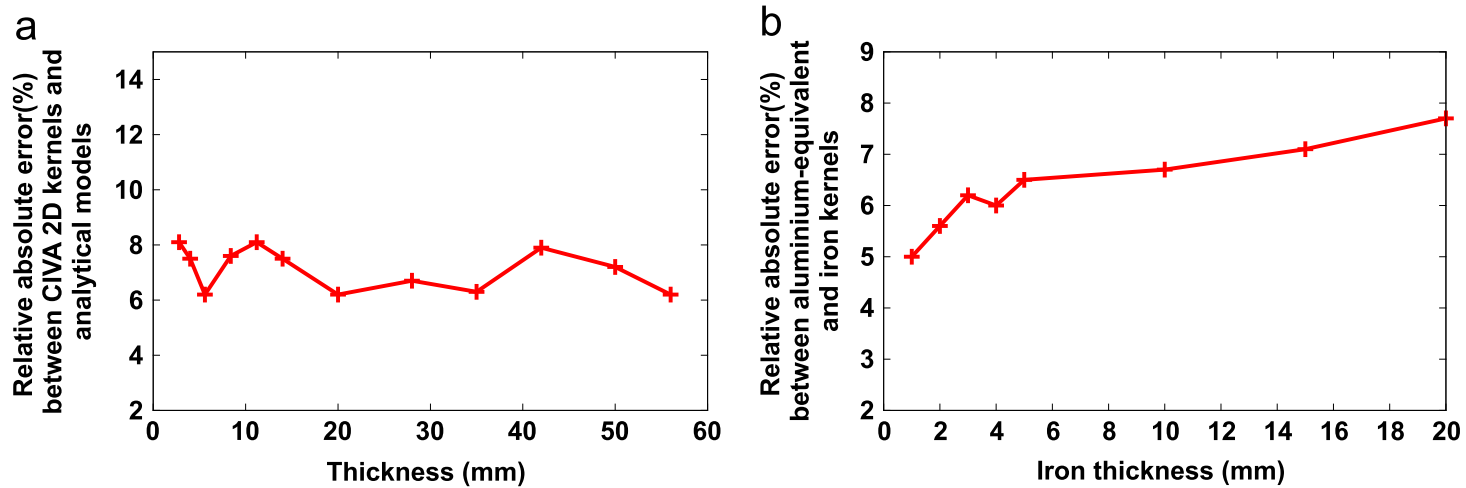

Fig. 6. Plot of relative absolute error with respect to thickness between (a) CIVA 2D kernels and analytical models, (b) aluminium-equivalent kernels and iron kernels.

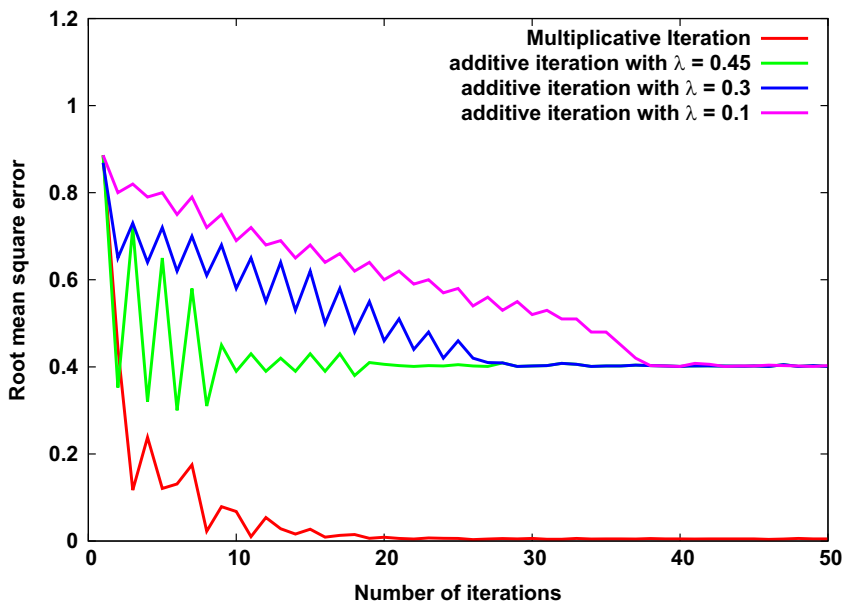

Fig. 7. Plot of error Vs number of iteration for different iteration schemes.

where $\rho_{e, A}$ and $\rho_{e, B}$ is the electron density for material A and $\mathrm{B}$ respectively. Given $\gamma$ be the ratio between these densities, we have

$\gamma=\frac{\rho_{e, A}}{\rho_{e, B}}=\frac{\rho_{A} Z_{A} A_{B}}{\rho_{B} Z_{B} A_{A}}$

where $\rho$ is the mass density, $Z$ is the atomic number and $A$ is mass number. Using Eqs. (11) and 12 we get

$T_{B}=\gamma T_{A}$

This property can be exploited for the scatter correction of heterogeneous objects. As the kernels for a particular thickness of a material do not depend on the atomic number of the material but only on its electron density. Kernels of single material can be used for the scatter correction of heterogeneous objects.

\subsection{Iterative scatter correction}

At high energies, the combined scatter of the object and the detector can be much higher than the true primary itself due to high SPR. In such case when a simple additive iteration approach is applied (Eq. (14)) to calculate the new primary estimate, negative values of the primary estimate can be obtained due to overestimation of scatter:

$P^{n+1}(m, n)=P^{n}(m, n)+\lambda\left(S^{n}(m, n)-S^{n+1}(m, n)\right)$

$\lambda$ represents the relaxation parameter. It is difficult in the first place to estimate the value of $\lambda$ that ensures positive primary estimate. Moreover, even if a suitable $\lambda$ value is chosen to ensure the first scatter estimate to be lower than the measured signal, this type of iteration scheme gives no guarantee that the primary estimate will be positive at every iteration step.

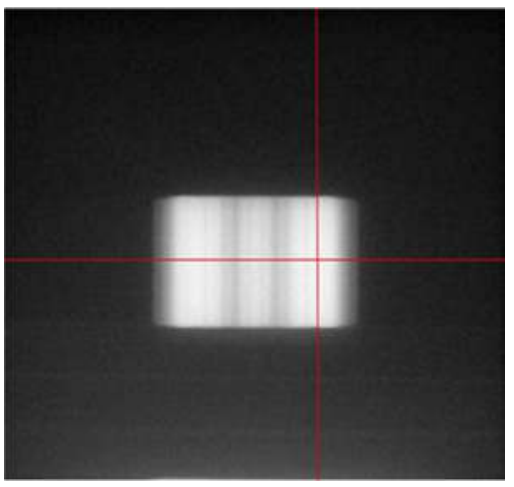

Fig. 8. Sample projection of iron hub sample.

Therefore we adopt a multiplicative iteration scheme given by Eq. (15) which ensures positive primary values.

The iterative scatter correction scheme consists of the following steps:

1. The measured projection is taken as the first estimate of the primary.

2. Equivalent thickness is calculated for each pixel by using Eq. (3).

3. Pixel wise convolution is performed by choosing the suitable kernel for the respective thicknesses.

4. Scatter is estimated either using Eq. (8) for the discrete approach or by using Eq. (10) for the continuous approach.

5. The primary estimate is updated using

$$
P^{n+1}(m, n)=P^{0}(m, n) \times \frac{P^{n}(m, n)}{P^{n}(m, n)+S^{n}(m, n)}
$$

Steps 1-5 are repeated until convergence is achieved.

\subsection{Validation on homogeneous object}

For the acquisition set up, the source to detector distance was kept $371 \mathrm{~cm}$ and the distance between source and axis of rotation was kept at $353 \mathrm{~cm}$. The set up mainly consisted of a X-ray source, an object rotational table and a flat panel detector.

The X-ray source unit used was a $400 \mathrm{kV}$ X-ray generator manufactured by Philips (model MG450). The maximum tube current was $15 \mathrm{~mA}$. Two focal spot sizes were available: $1.5 \mathrm{~mm}$ and $4.5 \mathrm{~mm}$ and angle of the target, made of tungsten, was $26^{\circ}$. A filtration of $4 \mathrm{~mm}$ of $\mathrm{Pb}+1 \mathrm{~mm}$ of $\mathrm{Cd}$ was used in order to reasonably remove the beam hardening effect. With this filtration the total number of X-ray photons with energy less than $200 \mathrm{keV}$ 
a

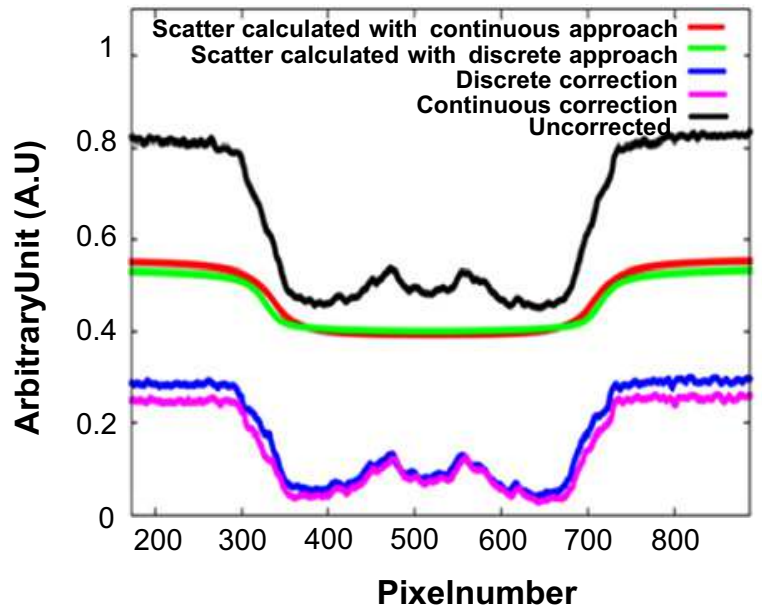

b

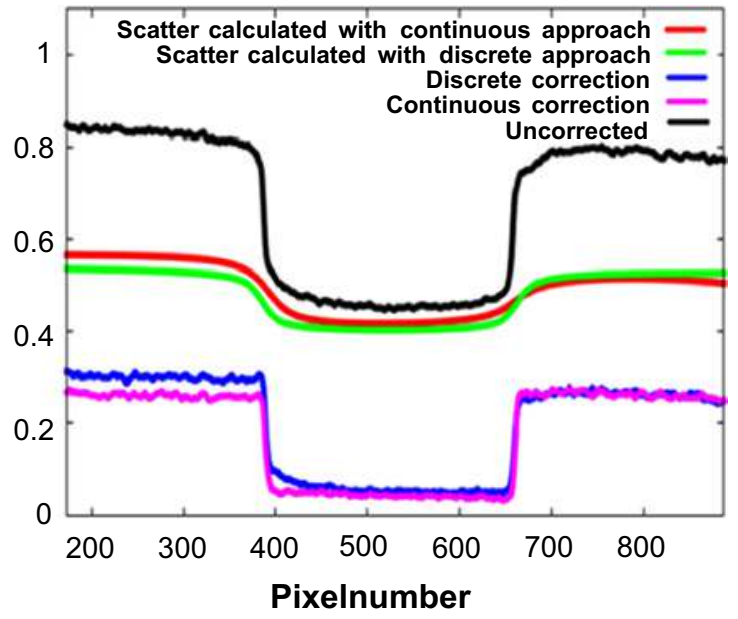

Fig. 9. (a) Horizontal profile. (b) Vertical profile of uncorrected and corrected projection by continuous and discrete method.

a

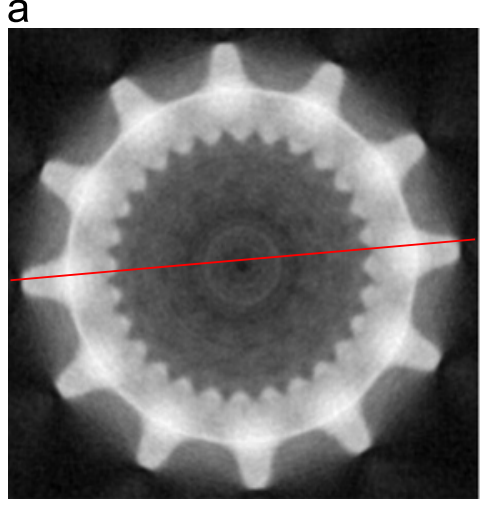

b

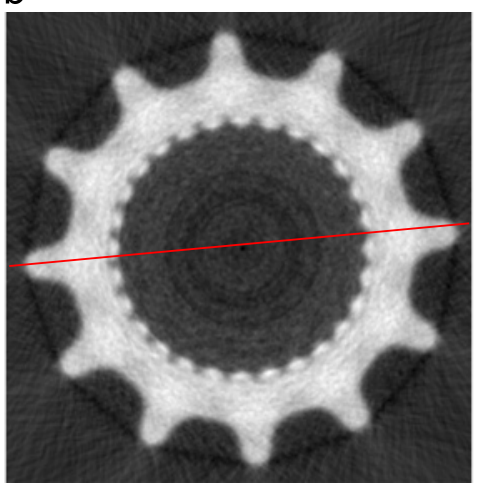

C

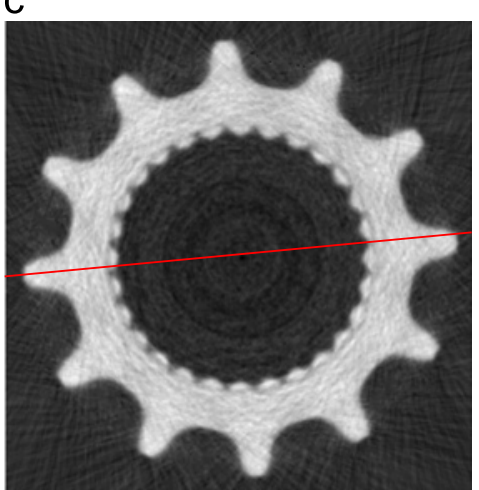

Fig. 10. Reconstruction slice of (a) Uncorrected projections, (b) Corrected projections by discrete method, (c) Corrected projections by continuous method.

Table 1

Mean and standard deviation values for aluminium and iron region for uncorrected and corrected reconstruction slices.

\begin{tabular}{llll}
\hline & & Mean $\left(\mathrm{cm}^{-1}\right)$ & Std. dev. \\
\hline Uncorrected & Air & 0.0240 & \pm 0.0022 \\
& Iron & 0.1960 & \pm 0.0068 \\
Discrete corrected & Air & 0.0028 & \pm 0.0056 \\
& Iron & 0.6340 & \pm 0.0046 \\
Continuous corrected & Air & 0.0012 & \pm 0.0059 \\
& Iron & 0.8000 & \pm 0.0086 \\
Mean spectrum value & Air & 0.0001 & \\
& Iron & 0.836 &
\end{tabular}

represented the $4 \%$ of the X-ray beam spectrum while it was $92 \%$ without filtration [5].

The flat panel detector called FlashScan 33 developed by Thales Electron Devices consisted of Gadolinium Oxysulfide (Tb) scintillator screen for the X-ray conversion. The detector was based on amorphous silicon TFTs array and was designed to work on a wide $\mathrm{kV}$ range (from $25 \mathrm{kV}$ to few $\mathrm{MeV}$ ). The active area consisted of 2240 $\times 3200$ pixels of $127 \mu \mathrm{m}$ size [5].

Acquisitions were performed on an iron hub sample as given in Fig. 1. The sample is $32 \mathrm{~mm}$ in height and $52 \mathrm{~mm}$ in the external diameter.

\subsection{Validation on heterogeneous object}

In order to check the feasibility of the algorithm for heterogeneous objects, projections were simulated in CIVA for an object, consisting of a $60 \mathrm{~mm}$ diameter cylinder of aluminum containing a $20 \mathrm{~mm}$ diameter cylinder of iron inspected at $450 \mathrm{keV}$ monochromatic source and a flat panel detector of $750 \times 750$ pixels with $220 \mu \mathrm{m}$ size. Fig. 2 shows the set up of the object.

\section{Results}

\subsection{Analytical fitting of kernels for continuous kernel map}

Fig. 3(a) shows the 1D profile of the kernels obtained by MC simulation in CIVA and 3(b) shows the kernels normalised to the maximum value of the detector kernel ( $0 \mathrm{~mm}$ thickness).

The fitting of parameters $\alpha, \beta, A, B, \sigma_{1}, \sigma_{2}$ with respect to thickness using least square fitting is shown in Fig. 4. The SPR first decreases and then increases for thicknesses above $20 \mathrm{~mm}$ due to higher attenuation of primary as seen in Fig. 3(a). Fig. 3(b), however, clearly shows that with increasing the thickness of the object the higher order scattering increases and broadens the shape of the kernels. Parameters $A$ and $B$, which are representing the amplitude of the shape of the kernels, first decrease up to a thickness of $20 \mathrm{~mm}$ and slowly increase beyond $20 \mathrm{~mm}$. For fitting this, we used Morse potential energy function. Parameter $\alpha$ which 


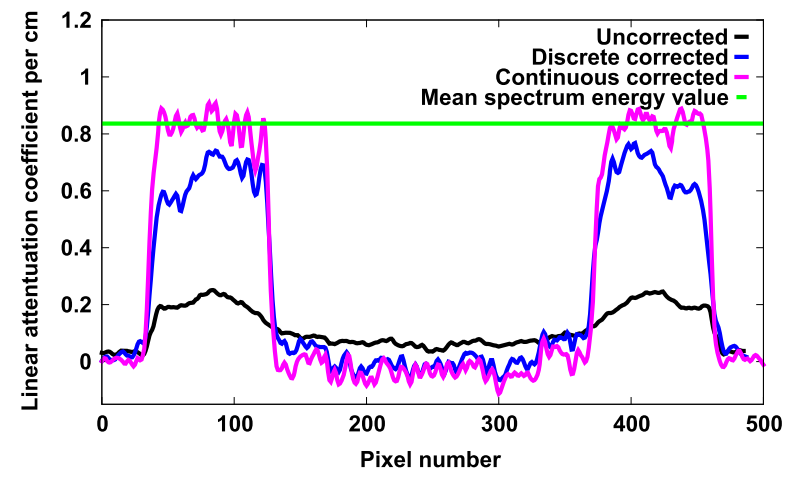

Fig. 11. Plot profile of the reconstruction slice of (a) Uncorrected projections, (b) Corrected projections by discrete method, (c) Corrected projections by continuous method.

is dependent on the attenuation, increases linearly with the thickness. Following the same argument parameter $\beta$, on the other hand, decreases with increasing the thickness. As mentioned in Section 2.2, $\sigma_{2}$ defining the scattering from the detector remains a constant with varying material thickness and $\sigma_{1}$ defining the higher order scattering from the object increases along with the thickness of the object. The relative absolute error with respect to thickness between CIVA 2D kernels and analytical models is between 6 and 8\% as shown in Fig. 6(a).

The kernels for aluminum and the equivalent thickness of iron using $\gamma=2.8$, computed from Eq. (12), for the geometry described in Section 2.6 are compared in Fig. 5 for different slab thicknesses. It can be seen from the comparison of the kernels (Fig. 5) that scatter to primary ratio is almost identical for aluminum slab and its equivalent thickness of iron slab. The relative absolute error with respect to thickness aluminium-equivalent kernels and iron kernels is between 5 and $8 \%$ as shown in Fig. 6(b). This allows us to evaluate the scatter correction of projections using only aluminum slab kernels. The results obtained are shown in Section 3.4.

\subsection{Multiplicative iteration vs additive iteration}

To check the efficiency of multiplicative iteration approach Vs additive iteration approach, we randomly initialized the scatter estimate maps $N$ ( $=50$ in our case) times for each type of iteration process. We then calculate the mean of the error in the convergence value from the true value, for these $N$ initial estimates at each iteration step. We plot the error Vs Number of iteration plot for multiplicative approach and additive approach for different values of $\lambda$. The result obtained is given in Fig. 7 .

In our case, we choose the maximum value of $\lambda=0.45$ to ensure that the first estimate of scatter is lower than the measured signal. From Fig. 7, it can be seen that additive approach is sensitive to the value of $\lambda$ converges with a higher error as compared to multiplicative scheme, due the presence of negative primary estimates within the iteration steps. The multiplicative approach, however, ensures positive primary estimate at each iteration step as well as converges with a faster rate (in this case iteration step 20).

\subsection{Scatter correction on homogeneous object}

Fig. 8 displays a sample projection of the iron-hub. To evaluate the performance of continuous and discrete scatter correction on the projections, the vertical and horizontal profiles of the corrected and uncorrected normalized projections are plotted in Fig. 9. Fig. 9 also displays calculated scatter profiles with discrete and continuous approach.

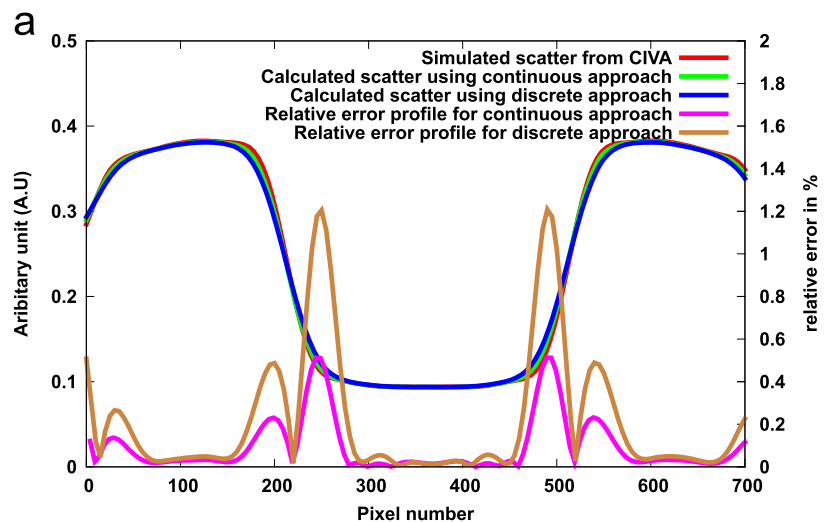

Plot profile of the scatter obtained using continuous and discrete approach

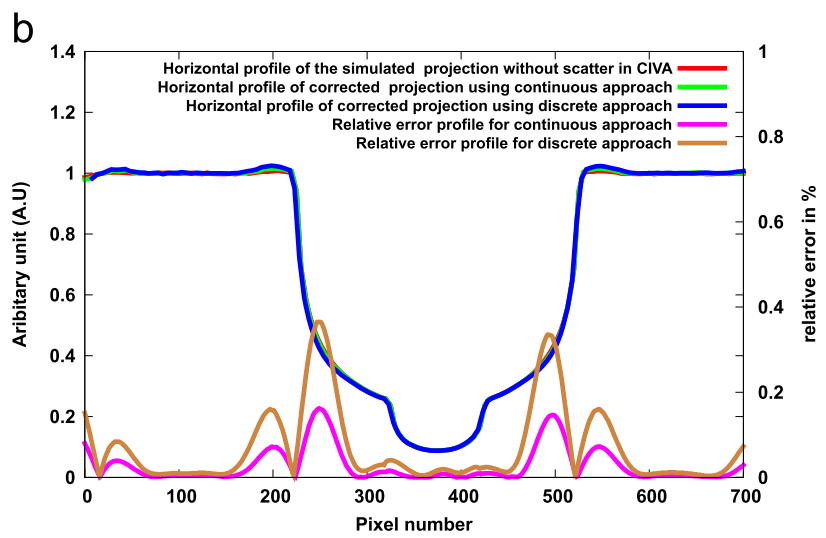

Horizontal profile of corrected projection using continuous and discrete approach

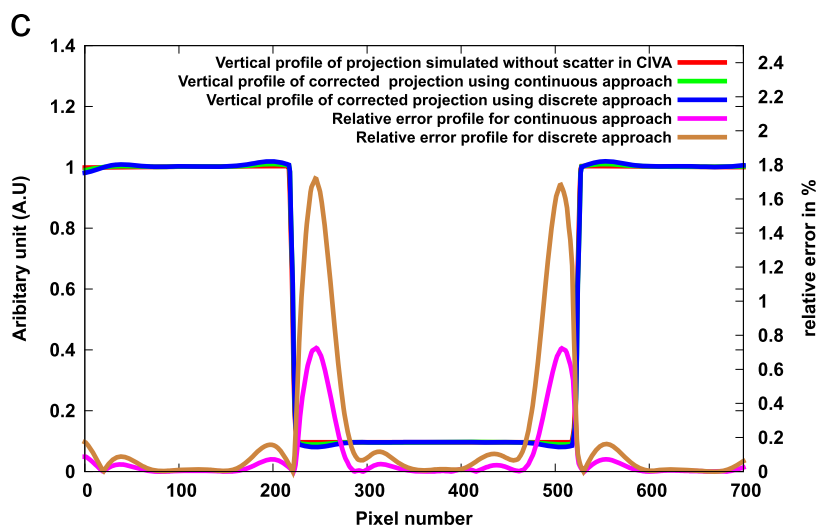

Vertical profile of corrected projection using continuous and discrete approach

Fig. 12. Plot profiles of scatter and corrected projections for the heterogeneous object. (a) Plot profile of the scatter obtained using continuous and discrete approach. (b) Horizontal profile of corrected projection using continuous and discrete approach. (c) Vertical profile of corrected projection using continuous and discrete approach.

On the vertical profile of corrected projection 9(b), we can see that the continuous method performs better edge enhancement of the object than the discrete method. This is due to better sampling of the kernels with respect to thickness at the edges.

Fig. 10 displays reconstruction slices of the top tip of the iron hub obtained for uncorrected and corrected projections using continuous and discrete methods. Table 1 displays the mean and standard deviation of the reconstructed values for corrected and 

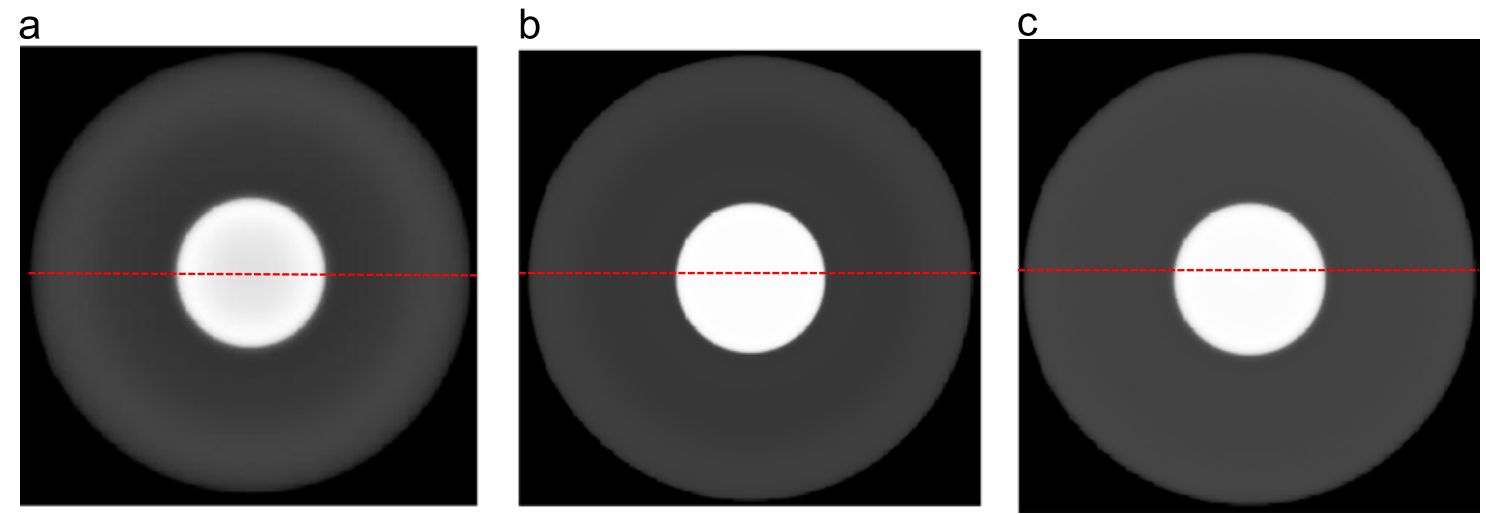

Fig. 13. Reconstruction slice of (a) Uncorrected projections. (b) Corrected projections by discrete method. (c) Corrected projections by continuous method.

Table 2

Mean and standard deviation values for aluminium and iron region for uncorrected and corrected reconstruction slices.

\begin{tabular}{lllll}
\hline & & $\begin{array}{l}\text { Mean } \\
\left(\mathrm{cm}^{-1}\right)\end{array}$ & Std. dev & $\begin{array}{l}\text { Relative absolute } \\
\text { error }(\%)\end{array}$ \\
\hline Uncorrected & Aluminium & 0.268 & \pm 0.0012 & 8.5 \\
& Iron & 0.560 & \pm 0.0058 & 22 \\
Discrete corrected & Aluminium & 0.252 & \pm 0.0090 & 2.0 \\
& Iron & 0.713 & \pm 0.0046 & 0.9 \\
Conitinuous & Aluminium & 0.249 & \pm 0.0074 & 0.8 \\
corrected & Iron & 0.718 & \pm 0.0042 & 0.2 \\
Theoretical values & Aluminium & 0.247 & & \\
& Iron & 0.720 & & \\
\hline
\end{tabular}

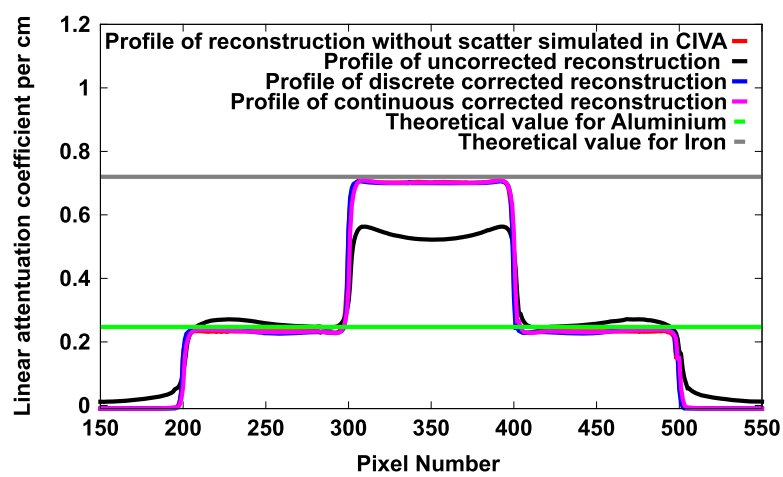

Fig. 14. Plot profile of the reconstruction slice obtained with (a) Projection without scatter from CIVA, (b) Uncorrected projections, (c) Corrected projections by discrete method, (d) Corrected projections by continuous method.

uncorrected data in air and iron region calculated using binary masks.

Uncorrected value of $0.196 \mathrm{~cm}^{-1}$ for the linear attenuation coefficient of iron is estimated by the discrete approach at $0.634 \mathrm{~cm}^{-1}$ whereas it is estimated at $0.80 \mathrm{~cm}^{-1}$ by the continuous approach. In the considered energy range, the value of linear attenuation constant per $\mathrm{cm}$ for mean energy $319 \mathrm{keV}$ is $0.836 \mathrm{~cm}^{-1}$, which is in agreement with the obtained result. The continuous method presents improved reconstruction values with respect to the discrete approach as can be seen in Fig. 11, which gives the plot profile of the reconstruction slice shown in Fig. 10, which exhibits better edge enhancement by the continuous approach.

The obtained result of the scatter profile and reconstruction values is in agreement with the correction performed using beam stop arrays method by Peterzol [5], which requires many acquisitions for the correction leading to higher dose.

\subsection{Scatter correction on heterogeneous object}

Fig. 12(a) shows the profile of the scatter obtained by simulation in CIVA. It is compared with the scatter profile obtained by the continuous and the discrete corrections. The relative error profiles of continuous scatter and discrete scatter with respect to the true value obtained from CIVA are also plotted. Clearly at the edges of the object continuous approach has lesser relative error as compared to discrete approach.

Fig. 12(b) and (c) show horizontal and vertical profile of the projection without scatter obtained in CIVA compared with corrected projection profile using the continuous and the discrete approach and their relative error profiles.

Fig. 13 displays reconstruction slices obtained for uncorrected and corrected projections using the continuous and the discrete method. Table 2 displays the mean and standard deviation of the reconstructed values for corrected and uncorrected data for aluminium and iron region.

The plot profile of the uncorrected and corrected reconstruction slices is shown in Fig. 14. The algorithm performs efficiently using only aluminum slab kernels. The linear attenuation coefficient obtained for iron and aluminum is $0.718 \mathrm{~cm}^{-1}$ and $0.249 \mathrm{~cm}^{-1}$ respectively for the continuous approach, which is in agreement with the expected linear attenuation value of $0.247 \mathrm{~cm}^{-1}$ for aluminium and $0.720 \mathrm{~cm}^{-1}$ for iron at $450 \mathrm{keV}$ monochromatic source.

\section{Discussion and conclusion}

Scatter Correction using pencil beam kernels produces significant improvement in the quantitative reconstruction values for the homogeneous object like iron hub. We adopted a continuous kernel approach by interpolating the kernels for each thickness. This approach offers superior improvement in the edges of the object after correction due to extended number of kernels. The reconstruction values are also improved in the continuous approach with respect to the discrete one especially for areas in the reconstruction slices where thin and thick structures are connected such as the tips of the iron hub.

We adopted a multiplicative iteration scheme which offers better convergence and ensures positive primary estimates at each iteration step as compared to additive iteration scheme which does not ensure positive primary estimates for higher SPR. As a result, multiplicative approach gives lesser error from the true value as compared to additive iteration approach.

The algorithm has been also tested for heterogeneous object. At the energy range used, Compton scattering is the most dominant interaction. The likelihood of interaction is proportional to electron density and is independent of the atomic number of the material. Therefore, 
kernels corresponding to a single material can be utilized for the correction of heterogeneous object projections. The obtained results are well within the expected reconstruction values proving the efficiency of the algorithm for heterogeneous objects.

\section{References}

[1] Schulze R, Heil U, Bruellmann D, Dranischnikow E, Schwanecke U, Schoemer E. Artefacts in CBCT: a review. Dentomaxillofac Radiol 2011;40(5):265-73.

[2] Shen S, Bloomquist A, Mawdsley G, Yaffe J, Elbakri I. Effect of scatter and an antiscatter grid on the performance of a slot-scanning digital mammography system. Med Phys 2006;33(4):1108-15.

[3] Krol A, Bassano D, Chamberlain C, Prasad S. Scatter reduction in mammography with air gap. Med Phys 1996;23(7):1263-70.

[4] Maltz J, Gangadharan B, Vidal M, Paidi A, Bose S, Faddego OMBA, et al. Focused beam-stop array for the measurement of scatter in megavoltage portal and cone beam ct imaging. Med Phys 2008;35(6):2452-62.
[5] Peterzol A, Letang JM, Babot D. A beam stop based correction procedure for high spatial frequency scatter in industrial cone-beam X-ray CT. NIMB 2008;266(18):4042-54.

[6] Zhao W, Zhu J, Wang L. Fast scatter artifacts correction for cone-beam ct without system modification and repeat scan. Med Phys, arXiv:1501.04409v1.

[7] Altunbas M, Shaw C, Chen L. A post-reconstruction method to correct cupping artifacts in cone beam breast computed tomography. Med Phys 2007; 34(7):3109-18.

[8] Hansen V, Swindell W, Evans P. Extraction of primary signal from epids using only forward convolution. Med Phys 1997;24(49):1477-84.

[9] Spies L, Ebert M, Groh BA, Hesse BM, Bortfeld T. Correction of scatter in megavoltage cone-beam CT. Phys Med Biol 2001;46:821-33.

[10] Maltz JS, Gangadharan B, Bose S, Hristov DH, Faddegona B, Paidi A, et al. Algorithm for $\mathrm{x}$-ray scatter beam-hardening and beam profile correction in diagnostic (kilovoltage) and treatment (megavoltage) cone beam CT. IEEE 2008;27(12):1791-810.

[11] Sun M, Star-Lack JM. Improved scatter correction using adaptive scatter kernel superposition. Phys Med Biol 2010;55(22):6675-720.

[12] 〈http://www-civa.cea.fr/〉.

[13] Tabary J, Guillemaud R, Mathy F, Glire A, Hugonnard P. Combination of high resolution analytically computed uncollided flux images with low resolution Monte Carlo computed scattered flux images. IEEE 2004;51(1):212-7. 